\title{
Analysis of lung tumor initiation and progression using conditional expression of oncogenic K-ras
}

\author{
Erica L. Jackson, ${ }^{1}$ Nicholas Willis, ${ }^{1}$ \\ Kim Mercer, ${ }^{1,2}$ Roderick T. Bronson, ${ }^{4}$ \\ Denise Crowley, ${ }^{1,2}$ Raymond Montoya, ${ }^{1}$ \\ Tyler Jacks, ${ }^{1,2,5}$ and David A. Tuveson ${ }^{1,2,3}$
}

${ }^{1}$ Department of Biology and ${ }^{2}$ Howard Hughes Medical Institute, Massachusetts Institute of Technology, Cambridge, Massachusetts 02139, USA; ${ }^{3}$ Dana-Farber Cancer Institute, Division of Adult Oncology, Boston, Massachusetts 02115, USA; ${ }^{4}$ Department of Pathology, Tufts University School of Medicine and Veterinary Medicine, Boston,

Massachusetts 02111, USA

\begin{abstract}
Adenocarcinoma of the lung is the most common form of lung cancer, but the cell of origin and the stages of progression of this tumor type are not well understood. We have developed a new model of lung adenocarcinoma in mice harboring a conditionally activatable allele of oncogenic $\mathrm{K}$-ras. Here we show that the use of a recombinant adenovirus expressing Cre recombinase (AdenoCre) to induce K-ras G12D expression in the lungs of mice allows control of the timing and multiplicity of tumor initiation. Through the ability to synchronize tumor initiation in these mice, we have been able to characterize the stages of tumor progression. Of particular significance, this system has led to the identification of a new cell type contributing to the development of pulmonary adenocarcinoma.
\end{abstract}

Received September 4, 2001; revised version accepted October 24, 2001.

Lung cancer is the leading cause of cancer deaths worldwide (Kerr 2001). Human lung cancers are categorized into four distinct histopathological classes: adenocarcinoma, squamous cell carcinoma, large cell carcinoma, and small cell carcinoma. The prevalence of adenocarcinoma is increasing, and adenocarcinoma is currently the most common type of lung cancer in the United States. Unlike squamous cell carcinoma, the stages of progression have not been well described for adenocarcinoma, and little is known about the cell type of origin or the characteristics of precursor lesions of pulmonary adenocarcinoma. Patients are usually diagnosed with lung cancer because of disease symptoms or incidental chest Xray findings. Perhaps owing to the inability to recognize premalignant lesions, patients are seldom diagnosed be-

[Key Words: K-ras; lung cancer; mouse models; Cre-loxP] ${ }^{5}$ Corresponding author.

E-MAIL tjacks@mit.edu; FAX (617) 253-9863.

Article and publication are at http://www.genesdev.org/cgi/doi/10.1101/ gad.943001. fore their cancer has reached an advanced stage (Tuveson and Jacks 1999).

Activating mutations of the K-ras oncogene are found in one-quarter to one-half of human lung adenocarcinomas. K-Ras is a membrane-associated GTPase signaling protein that regulates proliferation, differentiation, and cell survival (Campbell et al. 1998). Missense mutations at codons 12,13 , and 61 result in decreased GTPase activity and constitutive signaling. In the mouse, K-ras mutations are found in $>90 \%$ of spontaneous and chemically induced lung tumors (Malkinson 1998). In a previous effort to study spontaneous K-ras mutations in vivo, we constructed a novel mouse strain harboring a latent allele of K-ras G12D (referred to as K-ras ${ }^{L A}$ ) capable of spontaneous activation in vivo (Johnson et al. 2001). Kras $^{L A}$ mice develop a variety of tumor types, with $100 \%$ of the mice developing multiple early onset lung tumors. $K$-ras ${ }^{\mathrm{LA}}$ mice succumb at a young age from respiratory failure caused by an overwhelming number of predominantly early-stage lung lesions. This early mortality presumably limits the capacity for tumor progression. Also, in this and other mouse lung tumor models, the asynchrony of tumor development complicates analysis of tumor initiation and progression. Finally, the penetrance and multiplicity of tumor formation have been difficult to control, as some models have incomplete penetrance, but in others the number of primary lesions far exceeds the situation in humans. An ideal model would have one or a small number of tumors that could be followed over time through different stages of tumor progression.

In an effort to improve mouse lung tumor modeling, we used a Lox-Stop-Lox K-ras conditional mouse strain (referred to as LSL-K-ras G12D), in which expression of oncogenic $K$-ras is controlled by a removable transcriptional termination Stop element (Tuveson et al., in prep.). Floxed Stop elements have previously been used to suppress transcription of transgenic SV4O TAg (Lakso et al. 1992) as well as various reporter genes (Mao et al. 1999|. We have extended the use of conditional alleles to activate a gain-of-function mutation in a cellular oncogene. The endogenous K-ras locus is targeted in the $L S L$ K-ras G12D strain and, therefore, endogenous levels of oncogenic K-Ras G12D protein are expressed following removal of the Stop element. Removal of the Stop element from the $L S L$-K-ras G12D allele was achieved by the use of an AdenoCre, which allows control of the timing, location, and multiplicity of tumor initiation. Through the ability to synchronize tumor initiation, we have characterized the early stages of tumor progression. In addition, analysis of early-stage lesions has led to the discovery of a new cell type contributing to the development of pulmonary adenocarcinoma.

Results

Infection with high doses of AdenoCre causes excision of the transcriptional Stop element, resulting in expression of K-ras G12D and numerous lesions on the lung surface

Recombinant adenoviruses have been used as efficient vectors for gene delivery. Adenoviruses are able to infect a wide range of tissues and do not integrate into the host genome; expression of the delivered gene is therefore 
transient and there are no insertional mutations. To determine whether we could target K-ras G12D expression and subsequent tumor formation to the lungs, mice were infected with high doses of AdenoCre by intranasal instillation: $5 \times 10^{8} \mathrm{PFU}$ of virus that had been coprecipitated with $\mathrm{CaPO}_{4}$ was injected into the nasal passages of both $L S L$-K-ras $G 12 D$ and wild-type mice. The formation of coprecipitates of adenovirus and $\mathrm{CaPO}_{4}$ has been shown to increase the efficiency of infection of lung epithelium (Fasbender et al. 1998). Evidence from the $K$ ras $^{L A}$ mice indicated that numerous lesions could be observed on the surface of the lung within a month of birth (Johnson et al. 2001). Therefore, the infected LSL-K-ras G12D mice (hereafter referred to as Lox-K-ras G12D) were killed 4 wk postinfection. On examination, the lungs of the Lox-K-ras G12D mice had a cobblestone appearance, with numerous lesions on their surface. In contrast, the lungs from the wild-type mice appeared grossly normal (Fig. 1).

To confirm that the lesions were associated with excision of the transcriptional Stop element, genomic DNA was prepared from portions of the infected lungs, and PCR was performed to identify the presence of the activated K-ras allele. Only DNA prepared from the lungs of the Lox-Kras $G 12 D$ mice gave a product 40 bases larger than the wild-type allele, generated from the $K$ ras allele containing a single $\operatorname{lox} P$ site. The rearranged allele was not detected in any of the other tissues from the infected mice, indicating that infection and K-ras activation were limited to the lungs (data not shown). To verify that excision of the Stop element resulted in expression of K-RAS G12D protein, tissue extracts were analyzed by immunoprecipitation followed by Western blotting. Extracts were prepared from whole lungs 2 wh postinfection with $5 \times 10^{8}$ PFU of AdenoCre. Expression of the mutant protein was detected only in the lungs of Lox-K-ras G12D mice, and not in infected wild-type lungs or LSL-K-ras G12D lungs (data not shown; see Materials and Methods).

\section{Histological analysis of infected lungs reveals the presence of three distinct types of lesions}

Histological analysis of lungs taken at necropsy 6 wk postinfection revealed the presence of three distinct types of lesions: atypical adenomatous hyperplasia (AAH), epithelial hyperplasia $(\mathrm{EH})$ of the bronchioles, and adenomas. AAH is a proliferation of atypical epithelial cells growing along alveolar septae and does not disrupt the underlying lung architecture. The AAH present in the Lox-K-ras G12D mice closely resembles human AAH, a dysplastic lesion proposed to be a precursor of pulmonary adenocarcinoma (Kerr 2001). EH are papillary proliferations of epithelial cells lining the bronchioles; analogous lesions have not been identified in humans (nor was EH of the bronchiole seen in the K-ras ${ }^{L A}$ mouse model; Johnson et al. 2001). Adenomas are neoplasms with papillary, solid, or mixed architecture that distort or obliterate the alveolar septae. Adenomas of the lung are reported infrequently in humans, but this may reflect the fact that lung can- cer patients typically present with advanced disease. Interestingly, AAH lesions have been proposed to be adenoma-type lesions in the progression to human pulmonary adenocarcinoma (Kerr 2001). Therefore, the adenomas in the Lox-K-ras G12D mice may represent an intermediate stage between AAH and adenocarcinoma.

The adenomas in this model were predominantly of the papillary subtype, with solid and mixed solid/ papillary adenomas being much less frequent. A large proportion of the $\mathrm{AAH}$ and adenomas $(-80 \%)$ were continuous with $\mathrm{EH}$ of an adjacent respiratory bronchiole, raising the question of whether these are single lesions involving both the bronchiolar and alveolar epithelium or whether they are two adjacent lesions (see below.)

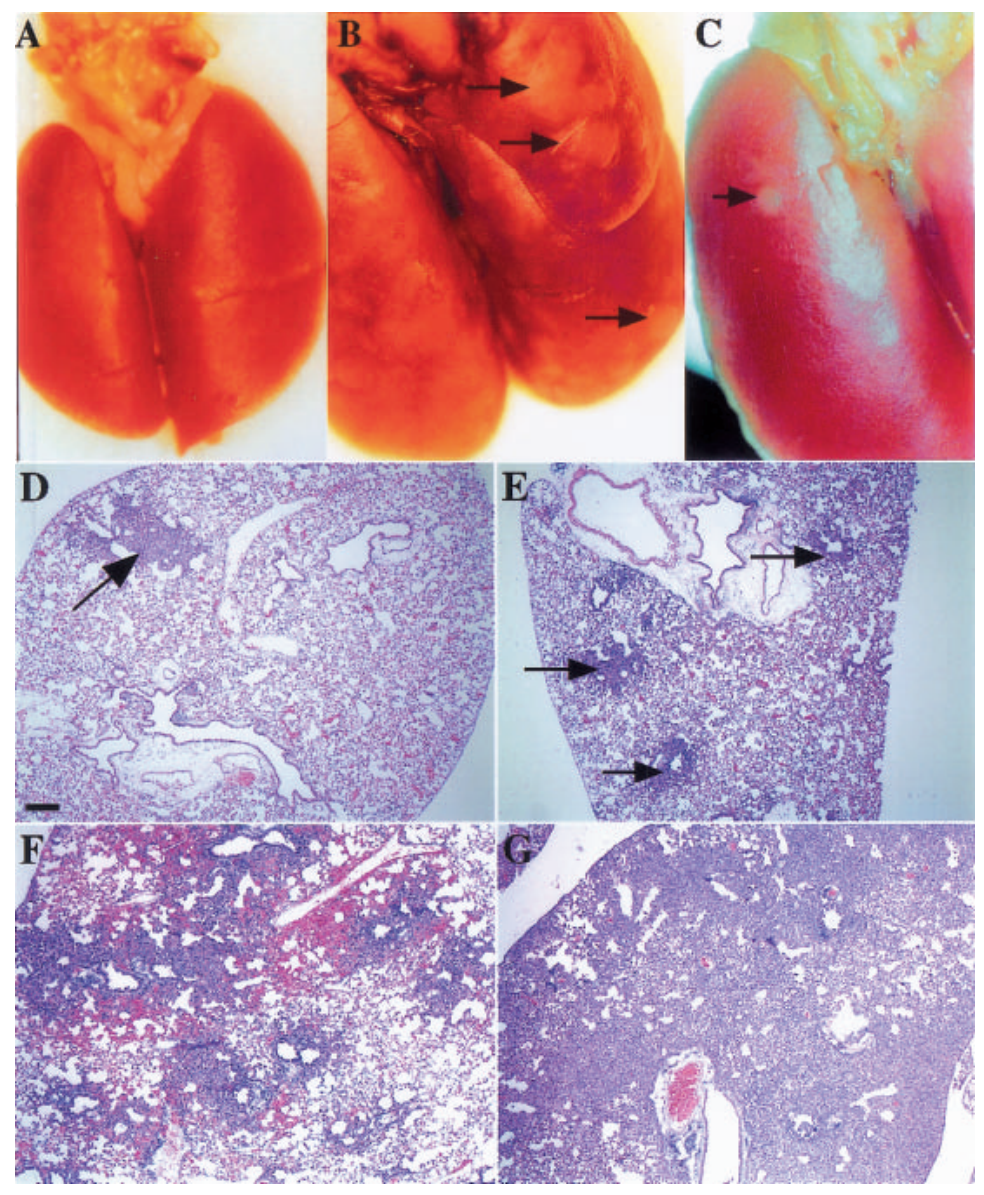

Figure 1. Viral dose dependence of tumor multiplicity. (A) Wild-type lungs 4 wk postinfection with $5 \times 10^{8} \mathrm{PFU}$ of AdenoCre. The surface of the lungs is smooth and uniform. (B) Lox-K-ras G12D lungs 4 wk postinfection with $5 \times 10^{8} \mathrm{PFU}$ of AdenoCre. The surface of the lungs has a cobblestone appearance; arrows indicate some of the lesions. (C) Lox-K-ras G12D lungs 10 wk postinfection with $5 \times 10^{5} \mathrm{PFU}$ of AdenoCre. The surface of the lungs is smooth with the exception of a single lesion indicated by an arrow. $(D)$ Histological sections of Lox-K-ras GD12D lungs 6 wk postinfection with $5 \times 10^{5} \mathrm{PFU}$, showing a single lesion (arrow). (E) Histological sections of Lox-K-ras GD12D lungs 6 wk postinfection with $5 \times 10^{6} \mathrm{PFU}$ showing several isolated lesions (arrows). (F) Histological sections of Lox-K-ras GD12D lungs 6 wk postinfection with $5 \times 10^{7}$ PFU showing areas of diffuse hyperplasia. $(G)$ Histological sections of Lox-K-ras GD12D lungs 6 wk postinfection with $5 \times 10^{8} \mathrm{PFU}$ showing diffuse hyperplasia. Scale bar indicates $200 \mu \mathrm{m}$. 
Tumor multiplicity can be controlled by varying the infectious dose

The large number of lesions seen on the surface of the lungs of the Lox-K-ras G12D mice infected with $5 \times 10^{8}$ PFU of virus indicated that this dose of virus might infect a large number of cells, resulting in an overwhelming lung tumor burden. Therefore, we performed a dose response study to address whether tumor multiplicity could be controlled by varying the amount of virus administered. Thus, 4-6 week old mice were infected with four doses of virus over a four-log range $\left(5 \times 10^{5}-5 \times 10^{8}\right.$ PFU) by intranasal instillation. Mice that received the two lower doses of virus contained well-isolated lesions that were readily quantifiable (Fig. 1A,B). However, specific lesions were not as easily identified in the lungs of mice infected with the two higher doses of virus owing to diffuse hyperplasia (Fig. 1C,D). A quantitative analysis was performed to analyze the tumor burden in lungs that received the two lower viral doses. As shown in Table 1, the number of lesions was directly proportional to the amount of virus used for infection. Of note, at the lowest dose of virus a median of 88 lesions was still observed. Therefore, lower doses of virus may result in still fewer tumors. The large standard deviation (SD) at this dose was attributable to two mice with unusually high tumor numbers. This may have been caused by differences in the efficiency of viral delivery, immune status, or other unknown factors. No lesions were observed in wild-type mice infected with any of the viral doses. Therefore, tumor multiplicity can be controlled by altering the amount of virus administered in the LSL-K-ras G12D model.

\section{Immunohistochemical analysis reveals hyperplasias are related to Clara cells and alveolar type II cells}

To investigate the histogenesis of the lung lesions, immunohistochemistry was performed using antibodies against Clara cell antigen (CCA or CC10) and the surfactant apoprotein-C (SP-C), commonly used markers that distinguish between Clara cells and alveolar type II cells, respectively. The isolated AAH lesions and adenomas were found to be positive for SP-C and negative for CCA, suggesting that these lesions arose from alveolar type II cells or their precursors (Fig. 2B). EH lesions of the bronchioles stained positively for CCA but were negative for SP-C, indicating the Clara cell lineage (Fig. 2A). Strikingly, however, the EH lesions that were continuous with AAH lesions or adenomas in serial sections revealed that some of the papillary structures located at the bronchiole/alveoli border stained positively for both SP-C and CCA (Fig. 2C,D).

Double immunofluorescence was performed to determine whether individual cells in these structures were expressing both SP-C and CCA. Cells expressing both SP-C and CCA were observed at the junction of bronchiolar and alveolar epithelium in the respiratory bronchioles (Fig. $2 \mathrm{~F}$ ), and in rare cases extended into the center of the adenoma. These double-positive cells com-
Table 1. Dose response study

\begin{tabular}{lcc}
\hline Viral dose & $\begin{array}{c}\text { Median tumor } \\
\text { number }\end{array}$ & SD \\
\hline $5 \times 10^{5} \mathrm{PFU}(n=6)$ & 87.5 & 253 \\
$5 \times 10^{6} \mathrm{PFU}(n=5)$ & 539 & 168 \\
\hline
\end{tabular}

Mice were sacrificed 6 wk postinfection. Lungs were step-sectioned at $100-\mu \mathrm{m}$ intervals and stained with H\&E. Those lesions readily identifiable under low-magnification were counted. The median tumor number $(n)$ of mice is shown.

prised a small percentage of the cells in the tumor; the majority of the cells were only SP-C positive. Doublepositive cells were not found in EH of larger bronchioles that do not contain alveoli in their walls, suggesting that this unusual expression pattern is not solely a consequence of Clara cell hyperplasia. Importantly, such

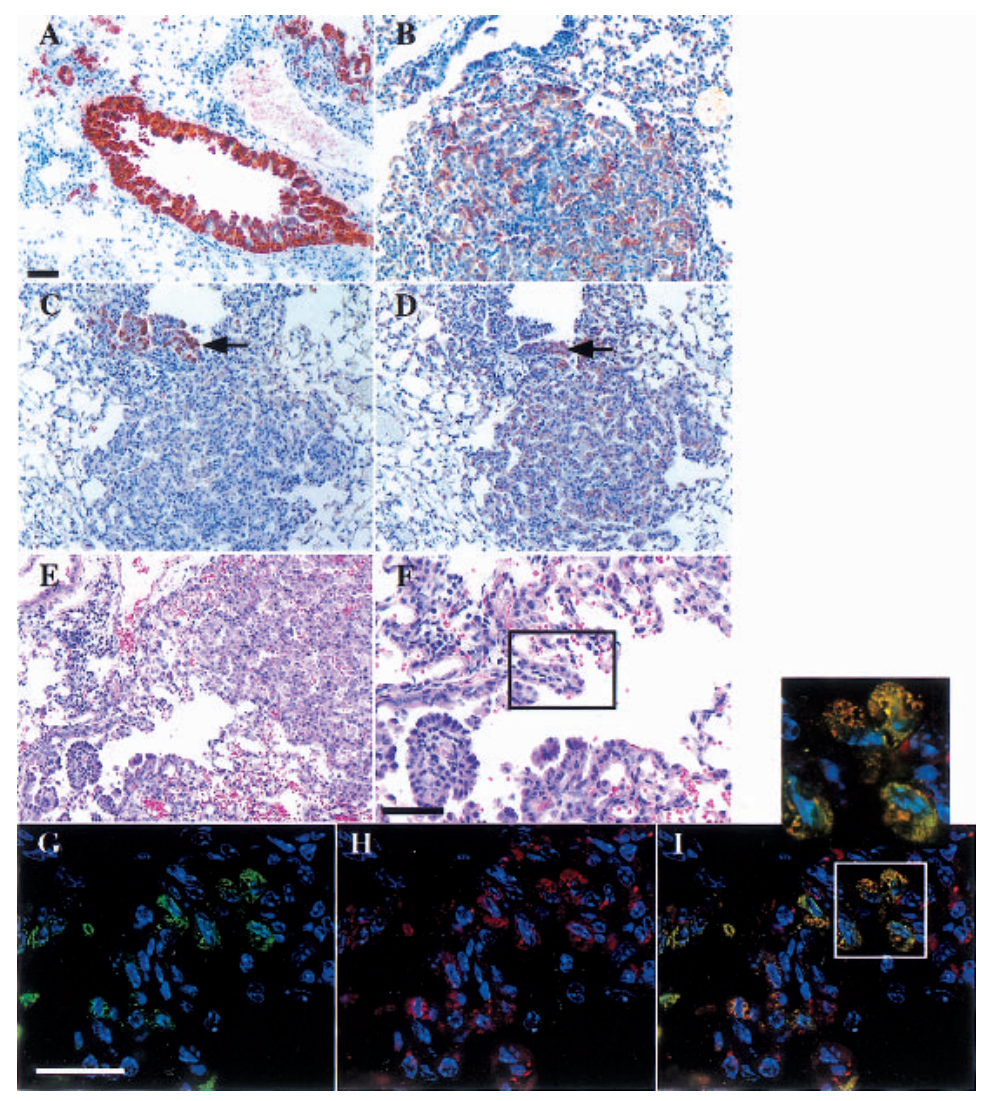

Figure 2. Immunophenotype of lesions in Lox-K-ras G12D mice. (A) CCApositive EH in a nonrespiratory bronchiole. (B) SP-C-positive adenoma (bottom) and SP-C-negative bronchiole (top left). (C) CCA-positive papillary structures in $\mathrm{EH}$ continuous with an adenoma. $(D)$ SP-C-positive papillary structures and adenoma in the same continuous lesion in the adjacent serial section. Arrows indicate the same papillary structure in $C$ and $D$ that is positive for both CCA and SP-C. (E) Histological section of EH continuous with an adenoma. $(F)$ Higher magnification of papillary structures in which SP-C/CCA double-positive cells are noted by double immunofluorescence on the adjacent serial section. The box indicates the area in which the double-positive cells shown in $G-I$ are located. $(G)$ CCA immunofluorescence alone. $(H)$ SP-C immunofluorescence alone. (I) Overlay of CCA and SP-C immunofluorescence showing the presence of cells expressing both CCA and SP-C. Scale bar indicates $50 \mu \mathrm{m}$ in $A-F$ and $25 \mu \mathrm{m}$ in $G-I$. 
double-positive cells were not detected in lungs from infected wild-type mice, nor were they observed in histologically normal respiratory bronchioles of Lox-K-ras G12D mice. These data provide evidence for the existence of a novel cell type, with characteristics of both Clara cells and alveolar type II cells, that may contribute to the formation of pulmonary adenomas. Furthermore, the existence of such cells supports the notion of tumor cell plasticity, in which pleuripotential tumor cells adopt different pseudodifferentiated states depending on their specific tissue microenvironment (see Discussion).

\section{Progression in the histological grade of the tumors is easily followed over time}

The histological changes that occur in the progression of lung adenocarcinomas from precursor lesions to malignant tumors have not been well characterized to date. Because the onset of K-ras G12D expression can be controlled in the LSL-K-ras G12D mice, tumor progression was examined in real time following the initiating event. To do so, we infected the $L S L$ - K-ras G12D mice with the two lower doses of virus $\left(5 \times 10^{5}\right.$ and $5 \times 10^{6}$ PFUs $)$ by intranasal instillation. Groups of mice were then killed at $2,6,12$, and $16 \mathrm{wk}$ postinfection. Histopathological analysis of lung lesions was performed on H\&E-stained sections at each time point. At 2 wk postinfection $(n=5)$, two types of lesions were detected: $\mathrm{AAH}$ and $\mathrm{EH}$ of the bronchioles (Fig. 3A,H). The EH lesions were often

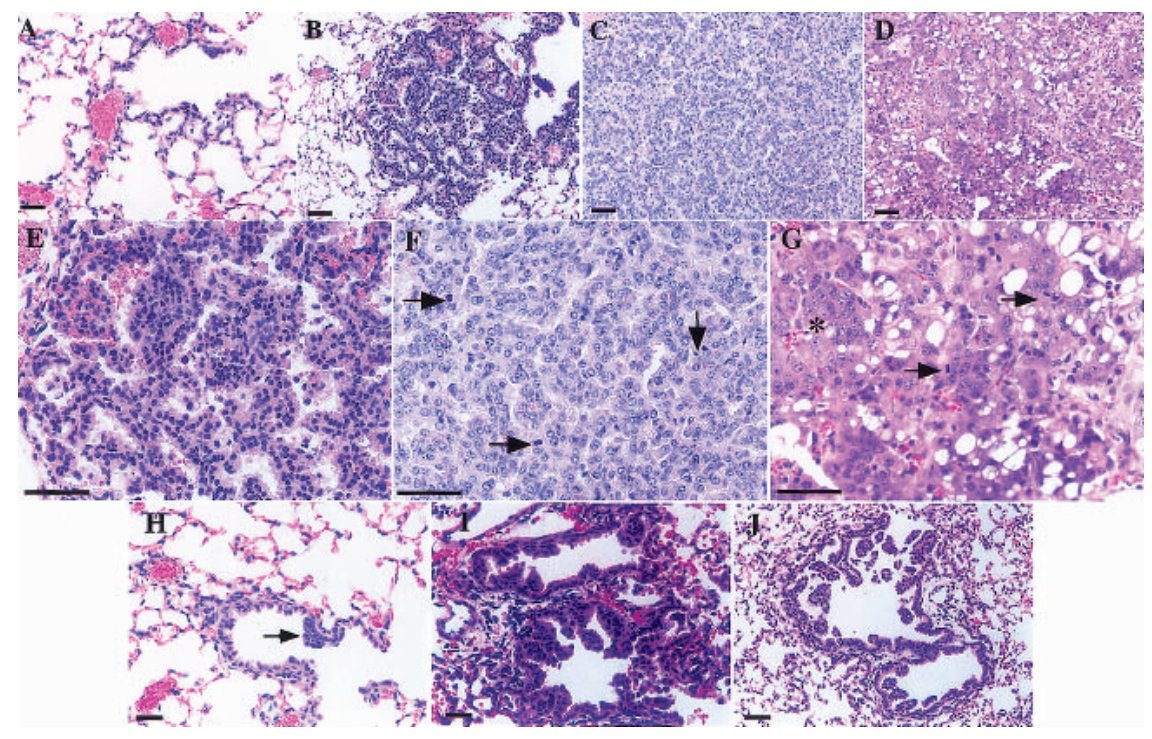

Figure 3. Time-course analysis of the stages of tumor progression in Lox-K-ras G12D mice. Mice were killed at $2(A, H), 6(B, E, I), 12(C, F, J)$, and $16(D, G)$ wk postinfection; histological sections of the lungs were examined for the presence of lesions. (A) Atypical adenomatous hyperplasia in Lox-K-ras G12D lungs 2 wk postinfection. (B) Papillary adenoma 6 wk postinfection. $(E)$ Higher magnification of the adenoma in $B .(C)$ Large adenoma 12 wk postinfection. $(F)$ Higher magnification of the lesion in $C$ (arrows indicate mitosis). (D) Adenocarcinoma 16 wk postinfection. (G) Higher magnification of the lesion in $D$. The tumor shows increased mitotic rate (arrows), nuclear enlargement, and prominent nucleoli (field of cells indicated by asterisk). $(H) \mathrm{EH}$ of a respiratory bronchiole 2 wk postinfection, showing focal hyperproliferation (arrow). (I) EH of a respiratory bronchiole 6 wk postinfection, with hyperproliferative cells in the alveolar compartment. (J) EH of a respiratory (top) and terminal (bottom) bronchiole 12 wk postinfection. continuous with AAH lesions situated in the alveolar space adjacent to the respiratory bronchioles. The apspread from the bronchioles, supported by the immunouorescence data described above.

At 6 wk postinfection, the lungs $(n=14)$ contained a AAH AH were larger and more cellular then those seen at 2 cult but sugesting that AAH lesions progress to ade mas. The majority of adenomas seen at this time were of papillary architecture. Some had well-circumscribed borders, whereas others extended out along the alveolar sepsisted at this time point (Fig. 3I), a subset of which again as well.

At 12 wk postinfection $(n=5)$, we observed the continued presence of $\mathrm{AAH}$; however, these lesions were outnumbered by adenomas. The adenomas were signifintly larger in size than those seen at the 6-wk time tures of malignancy including nuclear hyperchromatism and increased mitotic rate. $\mathrm{EH}$ of the bronchioles also persisted at this time (Fig. 3J). The lesions were more extensive and appeared to completely obstruct some bronchioles. In the continuous lesions, the underlying architecture of the bronchiole was often nearly completely destroyed, making it difficult to distinguish from the adenoma itself.

At 16 wk postinfection $(n=3)$, we observed the continued presence of large adenomas and $\mathrm{EH}$ of the bronchioles. In addition, overt adenocarcinomas were now present (Fig. 3D,G). The adenocarcinomas showed numerous mitoses (Fig. 3G, arrows) and nuclear pleomorphisms including enlarged nuclei with prominent nucleoli (Fig. 3G, asterisk). AAH lesions were rarely seen at this time point, indicating that these lesions are, indeed, precursor lesions that progress over time to tumors of more advanced stages.

\section{Discussion}

Here we report the creation of a new murine lung cancer model that overcomes many of the limitations of existing transgenic and carcinogen-induced models, and has enabled us to gain new insights into the initiation and early stages of pulmonary adenocarcinoma. A major limitation of existing murine lung cancer models is 
the inability to control tumor number. We have shown the ability to regulate the number of tumors by controlling the viral dose administered. It stands to reason that doses lower than $5 \times 10^{5}$ PFU will enable us to induce a very small number of tumors in these mice. This may more closely mimic human lung cancer, in which patients typically develop a single lung tumor that progresses over time to metastatic disease. In addition, the use of AdenoCre virus to induce expression of $K$-ras G12D has enabled us to synchronize tumor initiation. Because all of the lesions in a given mouse are induced simultaneously, we can follow the histological changes that occur during tumor progression. We cannot exclude the possibility that adenoviral infection or Cre expression may contribute to the tumorigenic process. However, none of the lungs of the infected wild-type mice showed any signs of hyperproliferation even at the highest viral doses, suggesting that there is little to no oncogenic stimulus inherent to the infection process.

The use of AdenoCre to synchronize tumor initiation has allowed a more careful examination of the events involved in the initiation of tumorigenesis. The short latency and high penetrance of AAH formation were particularly striking and suggested that expression of $K$-ras $G 12 D$ is sufficient for the induction of hyperplastic lesions. In humans, AAH is thought to correspond to one of the early stages in the development of pulmonary adenocarcinoma (Mori et al. 1993). However, it has proved difficult to study the progression of AAH in humans, as these microscopic lesions are usually identified as incidental findings in lungs resected for primary adenocarcinoma or at autopsy (Westra et al. 1996). The time-course study performed here indicated that $\mathrm{AAH}$ lesions became progressively larger and more cellular, until it became impossible to make a distinction between advanced AAH and adenoma. By 16 wk postinfection, AAH lesions were no longer seen, and overt adenocarcinomas were present. These findings provide strong evidence for a progression series from AAH to adenoma to adenocarcinoma.

The cell of origin of lung adenocarcinomas remains unknown, and candidates include multipotent stem cells, Clara cells, and alveolar type II cells (Dermer 1982). Human pulmonary adenocarcinomas are classified into six cytologic subtypes including both Clara cell type and alveolar type II cell type (Kobayashi et al. 1990), suggesting different cells of origin. However, ultrastructural and immunohistochemical studies have provided conflicting evidence. Alveolar type II cell markers are detected immunohistochemically in the majority of human adenocarcinomas, including those of the Clara cell subtype (Kitamura et al. 1997). Ultrastructural analysis of tumors has revealed the presence of single cells containing both lamellar bodies, characteristic of type II cells, and electron-dense Clara cell granules (Kitamura et al. 1997). However, the significance of these findings remains unclear, as it is difficult to distinguish Clara cell granules from lysosomes (Shimasoto 1994), and lamellated inclusions have been seen in normal mammalian Clara cells during development of the smooth endoplasmic reticulum (Plopper et al. 1983). Studies performed in animal models to address the cell of origin have also provided conflicting evidence. Transgenic mice expressing SV4O $\mathrm{TAg}$ from either the CC10 or SP-C promoter both develop adenomas (Wikenheiser et al. 1992; Magdaleno et al. 1997). Many carcinogen-induced mouse lung adeno- mas express SP-C or SP-A, markers used to identify type II cells (Mason et al. 2000), but some also show enzymatic activities characteristics of Clara cells (Gunning et al. 1991; Thaete and Malkinson 1991).

The double immunofluorescence experiments shown here clearly demonstrate the existence of SP-C/CCA double-positive cells within adenomatous lesions that are continuous with the bronchiolar epithelium. These findings shed new light on the somewhat ambiguous findings discussed above, and lead us to propose the existence of a pathway in the development of adenomas in which tumors arise from a cell type with characteristics of both Clara cells and type II cells. There are two possible models to describe this pathway. In the first model, oncogene activation induces hyperproliferation of Clara cells, which then undergo transdifferentiation as they move into the alveolar compartment. An alternative model is that these intermediate cells are the consequence of $K$-ras activation in a stem cell that has the potential to develop into either Clara cells or alveolar type II cells. Hyperplasia of such a cell type could give rise to both $\mathrm{EH}$ and adenomas. Coexpression of protein markers for Clara cells and type II cells is known to occur in epithelial progenitor cells during embryonic development in the mouse and other mammals (Wuenschell et al. 1996). However, no such stem cell has been identified in the adult lung.

The possibility of epithelial plasticity has been proposed to occur such that transformed Clara cells can transdifferentiate and take on characteristics of other pulmonary epithelial cells as influenced by the cellular environment (Wikenheiser and Whitsett 1997). Our data are consistent with this model. Double-positive cells are found exclusively at the bronchiolo-alveolar junction. They are situated at the end of a row of Clara cells and extend into the alveolar compartment. In addition, the SP-C/CCA double-positive cells show lower levels of CCA expression than their neighboring Clara cells. In light of this finding, we reexamined the tumors in the $K-$ ras $^{L A}$ mice, previously thought to arise from the alveolar type II cell lineage. Double-positive cells were found in adenomas and adenocarcinomas abutting bronchioles, located at the bronchiolo-alveolar border or in bronchioles surrounded by and continuous with tumor (data not shown). These findings suggest that the contribution of these intermediate cells may be a general phenomenon in K-ras-induced lung tumors.

Our findings are further supported by studies of bronchogenic carcinomas performed in both humans and dogs, in which proliferating type II cells were found in early lesions within the bronchial epithelium, which is usually devoid of this cell type (Ten Have-Opbroek et al. 1997). In dogs, carcinogen treatment of subcutaneous bronchial autografts (SBAs) resulted in adenocarcinomas comprised of alveolar type II cells (Ten HaveOpbroek et al. 1993). Because type II cells are not present in SBAs, the authors proposed the retrodifferentiation of neoplastic bronchiolar epithelial cells to a stemcell-like epithelium, which then gave rise to the type II cell adenocarcinomas. In light of these findings, the CCA/SP-C double-positive cells revealed in our immunofluorescence analysis may represent a retrodifferentiated cell type involved in the development of pulmonary tumors.

We have created a new murine model of lung cancer with many advantages over existing models. The ability 
to direct AdenoCre infection to other target organs in mice harboring the Lox-K-ras G12D and additional floxed alleles may be useful for the development of new organ-specific tumor models. In addition, the Lox-K-ras G12D model is particularly well suited for use in therapy and prevention studies, as the precise timing of tumor initiation can be controlled and tumor multiplicity can be adjusted.

\section{Materials and methods}

\section{Infection of lungs}

Mice were infected at 4-6 weeks of age. AdCre:CaPi coprecipitates were prepared as described (Fasbender et al. 1998). Mice were anesthetized with avertin. AdCre:CaPi coprecipitates were administered intranasally in two $62.5-\mu \mathrm{L}$ instillations. The second instillation was administered when breathing rates had returned to normal.

\section{Molecular and biochemical analysis}

For verification of Cre-mediated recombination, DNA was prepared from portions of lungs, tails, and kidneys. PCR was performed with primers flanking the Lox-Stop-Lox cassette (sequence available upon request). The K-ras and Lox-K-ras G12D alleles were detected, yielding a 265-bp and a 305-bp product, respectively.

For protein analysis of lungs by IP-Western, tissue lysates and immunoprecipitation were performed as described in Johnson et al. (1997). Western analysis was performed as described in Johnson et al. (2001).

\section{Histological analysis and immunohistochemistry}

Animals were killed at the times indicated and subjected to full necropsy. Histological and immunohistochemical analyses were performed as described in Johnson et al. (1997).

For immunofluorescence, sections were blocked at room temperature for $2 \mathrm{~h}$ in PBS containing $0.2 \%$ Triton X-100 and normal horse serum. They were then incubated at $4^{\circ} \mathrm{C}$ overnight with both rabbit polyclonal anti-CCA at 1:1000 and goat polyclonal anti-pro-SP-C (cat \#RDI-RTSURFCCabG) at 1:100 diluted in blocking buffer. Following incubation, sections were washed 3 times for 5 min with $0.2 \%$ Triton X-100 in PBS. Sections were then incubated at room temperature with rhodamine-conjugated anti-goat at a 1:200 dilution for $30 \mathrm{~min}$ followed by a 30-min incubation with FITC-conjugated anti-rabbit used at a 1:1000 dilution. Sections were then counterstained for $5 \mathrm{~min}$ with DAPI.

\section{Acknowledgments}

The CCA antibody was a generous gift from Anil Mukherjee from the NICHD/NIH. We thank Daniel Rines for help with deconvolution microscopy, and Ilona Linnoila and Margaret McLaughlin for helpful advice and critical reading of the manuscript. This work was funded in part by grants from the NCI. T.J. is an Associate Investigator of HHMI; D.A.T. is an HHMI Physician Postdoctoral Research Fellow.

The publication costs of this article were defrayed in part by payment of page charges. This article must therefore be hereby marked "advertisement" in accordance with 18 USC section 1734 solely to indicate this fact.

\section{References}

Campbell, S.L., Khosravi-Far, R., Rossman, K.L., Clark, G.J., and Der, C.J. 1998. Increasing complexity of Ras signaling. Oncogene 17: 13951413.

Dermer, G.B. 1982. Origin of bronchioloalveolar carcinoma and peripheral bronchial adenocarcinoma. Cancer 49: 881-887.

Fasbender, A., Lee, J.H., Walters, R.W., Moninger, T.O., Zabner, J., and Welsh, M.J. 1998. Incorporation of adenovirus in calcium phosphate precipitates enhances gene transfer to airway epithelia in vitro and in vivo. J. Clin. Invest. 102: 184-193.

Gunning, W.T., Stoner, G.D., and Goldblatt, P.J. 1991. Glyceraldehyde3-phosphate dehydrogenase and other enzymatic activity in normal mouse lung and in lung tumors. Exp. Lung Res. 17: 255-261.

Johnson, L., Greenbaum, D., Cichowski, K., Mercer, K., Murphy, E., Schmitt, E., Bronson, R.T., Umanoff, H., Edelmann, W., Kucher- lapati, R., et al. 1997. K-ras is an essential gene in the mouse with partial functional overlap with N-ras. Genes \& Dev. 11: 2468-2481.

Johnson, L., Mercer, K., Greenbaum, D., Bronson, R.T., Crowley, D., Tuveson, D.A., and Jacks, T. 2001. Somatic activation of the K-ras oncogene causes early onset lung cancer in mice. Nature 410: 11111116.

Kerr, K.M. 2001. Pulmonary preinvasive neoplasia. J. Clin. Pathol. 54: $257-271$.

Kitamura, H., Kameda, Y., Ito, T., Hayashi, H., Nakamura, N., Nakatani, Y., Inayama, Y., and Kanisawa, M. 1997. Cytodifferentiation of atypical adenomatous hyperplasia and bronchioloalveolar lung carcinoma: Immunohistochemical and ultrastructural studies. Virchows. Arch. 431: 415-424.

Kobayashi, T., Tsuda, H., Noguchi, M., Hirohashi, S., Shimosato, Y., Goya, T., and Hayata, Y. 1990. Association of point mutation in c-Ki-ras oncogene in lung adenocarcinoma with particular reference to cytologic subtypes. Cancer 66: 289-294.

Lakso, M., Sauer, B., Mosinger, B., Jr., Lee, E.J., Manning, R.W., Yu, S.H., Mulder, K.L., and Westphal, H. 1992. Targeted oncogene activation by site-specific recombination in transgenic mice. Proc. Natl. Acad. Sci. 89: 6232-6236.

Magdaleno, S.M., Wang, G., Mireles, V.L., Ray, M.K., Finegold, M.J., and DeMayo, F.J. 1997. Cyclin-dependent kinase inhibitor expression in pulmonary Clara cells transformed with SV40 large T antigen in transgenic mice. Cell Growth Differ. 8: 145-155.

Malkinson, A.M. 1998. Molecular comparison of human and mouse pulmonary adenocarcinomas. Exp. Lung Res. 24: 541-555.

Mao, X., Fujiwara, Y., and Orkin, S.H. 1999. Improved reporter strain for monitoring Cre recombinase-mediated DNA excisions in mice. Proc. Natl. Acad. Sci. 96: 5037-5042.

Mason, R.J., Kalina, M., Nielsen, L.D., Malkinson, A.M., and Shannon, J.M. 2000. Surfactant protein C expression in urethane-induced murine pulmonary tumors. Am. I. Pathol. 156: 175-182.

Mori, M., Chiba, R., and Takahashi, T. 1993. Atypical adenomatous hyperplasia of the lung and its differentiation from adenocarcinoma. Characterization of atypical cells by morphometry and multivariate cluster analysis. Cancer 72: 2331-2340.

Plopper, C.G., Alley, J.L., Serabjitsingh, C.J., and Philpot, R.M. 1983 Cytodifferentiation of the nonciliated bronchiolar epithelial (Clara) cell during rabbit lung maturation: An ultrastructural and morphometric study. Am. J. Anat. 167: 329-357.

Shimasoto. 1994. Pulmonary neoplasms. In Diagnostic surgical pathology, 3rd ed. (eds. S.S. Sternberg et al.), pp. 1045-1093. Raven Press, New York.

Ten Have-Opbroek, A.A., Hammond, W.G., Benfield, J.R., Teplitz, R.L., and Dijkman, J.H. 1993. Expression of alveolar type II cell markers in acinar adenocarcinomas and adenoid cystic carcinomas arising from segmental bronchi. A study in a heterotopic bronchogenic carcinoma model in dogs. Am. J. Pathol. 142: 1251-1264.

Ten Have-Opbroek, A.A., Benfield, J.R., van Krieken, J.H., and Dijkman, J.H. 1997. The alveolar type II cell is a pluripotential stem cell in the genesis of human adenocarcinomas and squamous cell carcinomas. Histol. Histopathol. 12: 319-336.

Thaete, L.G. and Malkinson, A.M. 1991. Cells of origin of primary pulmonary neoplasms in mice: Morphologic and histochemical studies. Exp. Lung Res. 17: 219-228.

Tuveson, D.A. and Jacks, T. 1999. Modeling human lung cancer in mice: Similarities and shortcomings. Oncogene 18: 5318-5324.

Westra, W.H., Baas, I.O., Hruban, R.H., Askin, F.B., Wilson, K., Offerhaus, G.J., and Slebos, R.J. 1996. K-ras oncogene activation in atypical alveolar hyperplasias of the human lung. Cancer Res. 56: 2224-2228.

Wikenheiser, K.A. and Whitsett, J.A. 1997. Tumor progression and cellular differentiation of pulmonary adenocarcinomas in SV40 large T antigen transgenic mice. Am. J. Respir. Cell Mol. Biol. 16: 713-723.

Wikenheiser, K.A., Clark, J.C., Linnoila, R.I., Stahlman, M.T., and Whitsett, J.A. 1992. Simian virus 40 large $T$ antigen directed by transcriptional elements of the human surfactant protein $\mathrm{C}$ gene produces pulmonary adenocarcinomas in transgenic mice. Cancer Res. 52: 5342-5352.

Wuenschell, C.W., Sunday, M.E., Singh, G., Minoo, P., Slavkin, H.C., and Warburton, D. 1996. Embryonic mouse lung epithelial progenitor cells co-express immunohistochemical markers of diverse mature cell lineages. J. Histochem. Cytochem. 44: 113-123. 


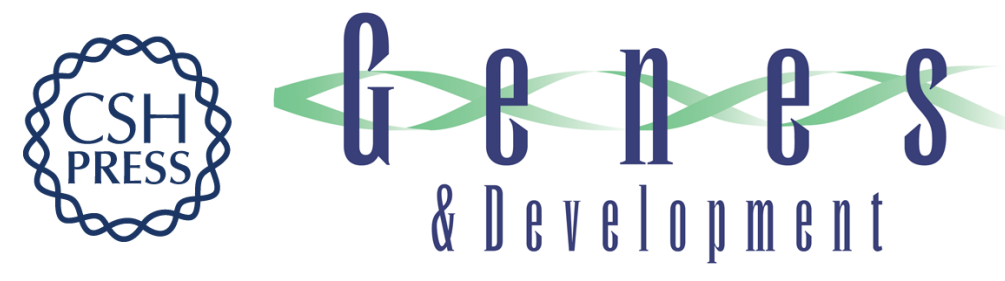

\section{Analysis of lung tumor initiation and progression using conditional expression of oncogenic K-ras}

Erica L. Jackson, Nicholas Willis, Kim Mercer, et al.

Genes Dev. 2001, 15:

Access the most recent version at doi:10.1101/gad.943001

References

This article cites 24 articles, 7 of which can be accessed free at:

http://genesdev.cshlp.org/content/15/24/3243.full.html\#ref-list-1

License

Email Alerting

Receive free email alerts when new articles cite this article - sign up in the box at the top

Service right corner of the article or click here.

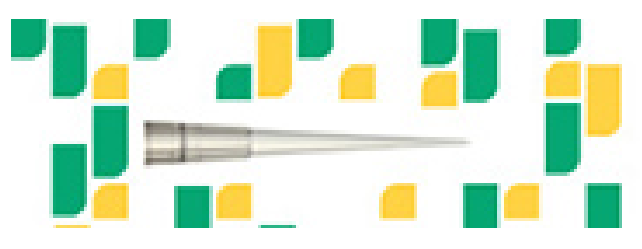

Focused on your science. 